\title{
Invariant measures for Markov operators with application to function systems
}

\author{
by \\ Tomasz SzareK (Katowice)
}

\begin{abstract}
A new sufficient condition for the existence of an invariant measure for Markov operators defined on Polish spaces is presented. This criterion is applied to iterated function systems.
\end{abstract}

0. Introduction. The theory of Markov processes is a fast developing topic which has been extensively studied during the last few years. The reason for this study was the progress in the theory of fractals. Markov processes can be considered from two points of view. They can be investigated by purely probabilistic and purely analytic methods. In our paper we use the second approach. More precisely, let $\left\{Z_{n}\right\}$ be a homogeneous Markov chain taking values in a metric space $(X, \varrho)$ and let $\pi$ be its transition kernel, i.e.

$$
\operatorname{Prob}\left(Z_{n+1} \in A \mid Z_{n}=x_{n}, \ldots, Z_{0}=x_{0}\right)=\pi\left(x_{n}, A\right)
$$

for all $n \in \mathbb{N}$ and Borel sets $A$. It is of great interest to give sufficient conditions for the existence of an invariant probability measure for the Markov chain $\left\{Z_{n}\right\}$, i.e. a probability measure $\mu_{*}$ satisfying

$$
\mu_{*}(A)=\int_{X} \pi(x, A) \mu_{*}(d x)
$$

for all Borel sets $A$ (see $[4,9,13-16])$.

In our analytic approach we consider the Markov operator $P$ defined on the space of all Borel measures given by

$$
P \mu(A)=\int_{X} \pi(x, A) \mu(d x) .
$$

Consequently, searching for invariant measures of Markov processes can be replaced by searching for fixed points of $P$. Using this method does not involve the probabilistic background of the transition kernels $\pi$.

2000 Mathematics Subject Classification: Primary 47A35; Secondary 28D15.

Key words and phrases: Markov operator, iterated function system, invariant measure. 
The theory of Markov operators $P$ with $X$ compact is well developed (see [5]). For example, the proof of the existence of an invariant measure goes as follows. First we construct a positive invariant functional on the space of all bounded continuous functions $f: X \rightarrow \mathbb{R}$ and then using the Riesz representation theorem we define an invariant measure. This method was extended by A. Lasota and J. Yorke to the case when $X$ is a locally compact $\sigma$-compact metric space [12]. When $X$ is any Polish space this idea breaks down, since a positive functional may not correspond to a measure. In our study we base on the concept of tightness (see also [19]).

In the second part of our paper we consider Markov processes generated by iterated function systems. Recently such systems have been studied in detail because of their close connection to fractals $[1,2,10]$ and semifractals [11]. We investigate iterated function systems with place dependent probabilities extending the well known theorem due to Barnsley et al. [2] to Polish spaces. This part of the paper also generalizes our earlier results [18, 19].

The outline of the paper is as follows. Section 1 contains some notation and definitions from the theory of Markov operators. In this section we also introduce the definition of semi-concentrating Markov operators. Such operators are studied in Section 2. We prove there the main results of the paper-Theorems 2.1 and 2.2 which assure, for semi-concentrating Markov operators, the existence of an invariant measure and the attractiveness of iterates to the family of measures supported on some compact set.

In Section 3 we introduce iterated function systems and discuss the problem of stability.

1. Preliminaries. Let $(X, \varrho)$ be a Polish space, i.e. a separable, complete metric space. Throughout this paper $B(x, r)$ stands for the closed ball in $X$ with centre at $x$ and radius $r$. For every $C \subset X$ and $r>0$ we denote by $\mathcal{N}^{0}(C, r)$ the open $r$-neighbourhood of $C$, i.e.

$$
\mathcal{N}^{0}(C, r)=\{x \in X: \varrho(C, x)<r\}
$$

and by $\mathcal{N}(C, r)$ the closed $r$-neighbourhood of $C$, i.e.

$$
\mathcal{N}(C, r)=\{x \in X: \varrho(C, x) \leq r\},
$$

where $\varrho(C, x)=\inf \{\varrho(x, y): y \in C\}$. Moreover, $\operatorname{diam} C$ is the diameter of $C$, i.e. $\operatorname{diam} C=\sup \{\varrho(x, y): x, y \in C\}$.

Let $\mathcal{B}(X)$ and $\mathcal{B}_{\mathrm{b}}(X)$ denote the families of all Borel sets and all bounded Borel sets in $X$, respectively. We denote by $\mathcal{C}(\varepsilon), \varepsilon>0$, the family of all $C \in \mathcal{B}(X)$ for which there exists a finite set $\left\{x_{1}, \ldots, x_{n}\right\} \subset X$ such that $C \subset \bigcup_{i=1}^{n} B\left(x_{i}, \varepsilon\right)$, and by $\mathcal{C}^{k}(\varepsilon), \varepsilon>0, k \in \mathbb{N}$, the family of all $C \in \mathcal{C}(\varepsilon)$ such that $C \subset \bigcup_{i=1}^{k} B\left(x_{i}, \varepsilon\right)$ for some $\left\{x_{1}, \ldots, x_{k}\right\} \subset X$. 
Let $\mathcal{M}_{\text {fin }}$ and $\mathcal{M}_{1}$ denote the sets of Borel measures (nonnegative, $\sigma$ additive) on $X$ such that $\mu(X)<\infty$ and $\mu(X)=1$, respectively. The elements of $\mathcal{M}_{1}$ are called distributions. We denote by $\mathcal{M}_{\text {sig }}$ the family of all signed measures:

$$
\mathcal{M}_{\text {sig }}=\left\{\mu_{1}-\mu_{2}: \mu_{1}, \mu_{2} \in \mathcal{M}_{\text {fin }}\right\} .
$$

We say that $\mu \in \mathcal{M}_{\text {fin }}$ is concentrated on $A \in \mathcal{B}(X)$ if $\mu(X \backslash A)=0$; let $\mathcal{M}_{1}^{A}$ denote the set of all distributions concentrated on $A \in \mathcal{B}(X)$.

For $A \subset X$ the symbol $\mathbf{1}_{A}$ stands for the characteristic function of $A$. As usual, $B(X)$ is the space of all bounded Borel measurable functions $f: X \rightarrow \mathbb{R}$ and $C(X)$ the subspace of all bounded continuous functions. In both spaces the norm is

$$
\|f\|_{0}=\sup _{x \in X}|f(x)| .
$$

To simplify the notation we write

$$
\langle f, \nu\rangle=\int_{X} f(x) \nu(d x) \quad \text { for } f \in B(X), \nu \in \mathcal{M}_{\text {sig }} .
$$

In $\mathcal{M}_{\text {sig }}$ we introduce the Fortet-Mourier norm (see [8]):

$$
\|\nu\|=\sup \{|\langle f, \nu\rangle|: f \in F\},
$$

where $F$ consists of all functions $f \in C(X)$ such that $\|f\|_{0} \leq 1$ and $\mid f(x)-$ $f(y) \mid \leq \varrho(x, y)$.

It is known that the convergence $\lim _{n \rightarrow \infty}\left\|\mu_{n}-\mu\right\|=0$ for $\mu_{n}, \mu \in \mathcal{M}_{1}$ is equivalent to the weak convergence of $\left(\mu_{n}\right)_{n \geq 1}$ to $\mu$ (see [6]).

Let $\Theta \subset \mathcal{M}_{1}$. We say that $\Theta$ is tight if for every $\varepsilon>0$ there exists a compact set $K \subset X$ such that $\mu(K) \geq 1-\varepsilon$ for all $\mu \in \Theta$. It is well known (see [3]) that if the family $\left(\mu_{n}\right)_{n \geq 1}$ of distributions is tight then there exists a subsequence $\left(m_{n}\right)_{n \geq 1}$ of integers and a measure $\mu_{*} \in \mathcal{M}_{1}$ such that

$$
\lim _{n \rightarrow \infty}\left\|\mu_{m_{n}}-\mu_{*}\right\|=0 \text {. }
$$

An operator $P: \mathcal{M}_{\text {fin }} \rightarrow \mathcal{M}_{\text {fin }}$ is called a Markov operator if it satisfies the following two conditions:

(i) positive linearity: $P\left(\lambda_{1} \mu_{1}+\lambda_{2} \mu_{2}\right)=\lambda_{1} P \mu_{1}+\lambda_{2} P \mu_{2}$ for $\lambda_{1}, \lambda_{2} \geq 0$ and $\mu_{1}, \mu_{2} \in \mathcal{M}_{\text {fin }}$,

(ii) preservation of the norm: $P \mu(X)=\mu(X)$ for $\mu \in \mathcal{M}_{\text {fin }}$.

Every Markov operator can be extended to the space of all signed measures. Namely for every $\nu \in \mathcal{M}_{\text {sig }}, \nu=\mu_{1}-\mu_{2}$, we set

$$
P \nu=P \mu_{1}-P \mu_{2} \text {. }
$$

A linear operator $U: B(X) \rightarrow B(X)$ is called dual to $P$ if

$$
\langle U f, \mu\rangle=\langle f, P \mu\rangle \quad \text { for } f \in B(X), \mu \in \mathcal{M}_{\text {fin }} .
$$


Setting $\mu=\delta_{x}$ in (1.1) we obtain

$$
U f(x)=\left\langle f, P \delta_{x}\right\rangle \quad \text { for } f \in B(X), x \in X,
$$

where $\delta_{x} \in \mathcal{M}_{1}$ is the point (Dirac) measure supported at $x$.

From (1.1) it follows immediately that $U$ is a linear operator satisfying

$$
\begin{array}{cl}
U f \geq 0 & \text { for } f \geq 0, f \in B(X), \\
& U \mathbf{1}_{X}=\mathbf{1}_{X}, \\
U f_{n} \downarrow 0 \quad \text { for } f_{n} \downarrow 0, f_{n} \in B(X) .
\end{array}
$$

Conditions (1.3)-(1.5) allow one to reverse the roles of $P$ and $U$. Namely we define an operator $P: \mathcal{M}_{\text {fin }} \rightarrow \mathcal{M}_{\text {fin }}$ by setting, for $A \in \mathcal{B}(X)$,

$$
P \mu(A)=\left\langle U \mathbf{1}_{A}, \mu\right\rangle \quad \text { for } \mu \in \mathcal{M}_{\text {fin }} .
$$

Assume now that $P$ and $U$ are given. If $f: X \rightarrow[0, \infty)$ is a Borel measurable function, not necessarily bounded, we may assume that

$$
U f(x)=\lim _{n \rightarrow \infty} U f_{n}(x),
$$

where $\left(f_{n}\right)_{n \geq 1}, f_{n} \in B(X)$, is an increasing sequence of functions converging pointwise to $f$. From the Lebesgue monotone convergence theorem it follows that $U f$ satisfies (1.1).

A Markov operator $P$ is called a Feller operator if there exists an operator $U: B(X) \rightarrow B(X)$ satisfying (1.1) such that

$$
U f \in C(X) \quad \text { for } f \in C(X) \text {. }
$$

A Markov operator $P$ is called nonexpansive if

$$
\left\|P \mu_{1}-P \mu_{2}\right\| \leq\left\|\mu_{1}-\mu_{2}\right\| \quad \text { for } \mu_{1}, \mu_{2} \in \mathcal{M}_{1} \text {. }
$$

Let $P$ be a Markov operator. A measure $\mu \in \mathcal{M}_{\text {fin }}$ is called stationary or invariant if $P \mu=\mu$, and $P$ is called asymptotically stable if there exists a stationary distribution $\mu_{*}$ such that

$$
\lim _{n \rightarrow \infty}\left\|P^{n} \mu-\mu_{*}\right\|=0 \quad \text { for } \mu \in \mathcal{M}_{1} .
$$

Clearly the distribution $\mu_{*}$ satisfying (1.9) is unique.

We introduce the following notation:

$$
\omega(\mu)=\left\{\nu \in \mathcal{M}_{1}: \exists\left\{m_{n}\right\}_{n \geq 1}, m_{n} \rightarrow \infty, P^{m_{n}} \mu \rightarrow \nu\right\} .
$$

An operator $P$ is called globally concentrating if for every $\varepsilon>0$ and $A \in \mathcal{B}_{\mathrm{b}}(X)$ there exist $Y \in \mathcal{B}_{\mathrm{b}}(X)$ and $n_{0} \in \mathbb{N}$ such that

$$
P^{n} \mu(Y) \geq 1-\varepsilon \quad \text { for } n \geq n_{0}, \mu \in \mathcal{M}_{1}^{A} \text {. }
$$

An operator $P$ is called semi-concentrating if for every $\varepsilon>0$ there exist $C \in \mathcal{C}(\varepsilon)$ and $\alpha>0$ such that

$$
\liminf _{n \rightarrow \infty} P^{n} \mu(C)>\alpha \quad \text { for } \mu \in \mathcal{M}_{1} \text {. }
$$


2. The existence of invariant measures. We start with easy lemmas:

Lemma 2.1. If $\left\|\mu_{1}-\mu_{2}\right\| \leq \varepsilon^{2}$ for $\mu_{1}, \mu_{2} \in \mathcal{M}_{1}$ and some $\varepsilon>0$ then

$$
\mu_{1}\left(\mathcal{N}^{0}(C, \varepsilon)\right) \geq \mu_{2}(C)-\varepsilon \quad \text { for } C \in \mathcal{B}(X) .
$$

Lemma 2.2. Let $P$ be a nonexpansive Markov operator. Assume that there exists a measure $\mu \in \mathcal{M}_{1}$ such that for every $\varepsilon>0$ there is a set $C \in \mathcal{C}(\varepsilon)$ satisfying $P^{n} \mu(C) \geq 1-\varepsilon$ for $n \in \mathbb{N}$. Then $\left(P^{n} \mu\right)_{n \geq 1}$ is tight. Moreover, $P$ has an invariant distribution.

The proofs of Lemmas 2.1 and 2.2 can be found in [19].

Let $P$ be a Markov operator. We denote by $\mathcal{T}(\varepsilon), \varepsilon>0$, the family of all $C \in \mathcal{C}(\varepsilon)$ such that there exists a positive number $\alpha$ satisfying

$$
\liminf _{n \rightarrow \infty} P^{n} \mu(C)>\alpha \quad \text { for } \mu \in \mathcal{M}_{1} \text {. }
$$

For $\varepsilon>0$ and $k \in \mathbb{N}$ write

$$
\mathcal{T}^{k}(\varepsilon)=\mathcal{C}^{k}(\varepsilon) \cap \mathcal{T}(\varepsilon) .
$$

We are now in a position to formulate the following technical lemma:

Lemma 2.3. Let $P$ be a nonexpansive and semi-concentrating Markov operator. Then for every $\varepsilon>0$ there exist an integer $k$, a sequence $\left(A_{1}, \ldots, A_{k}\right)$ with $A_{i} \in \mathcal{B}(X), \operatorname{diam} A_{i} \leq \varepsilon$ for $i=1, \ldots, k$, and a measure $\mu_{0} \in \mathcal{M}_{1}$ such that $\bigcup_{i=1}^{k} A_{i} \in \mathcal{T}^{k}(\varepsilon)$ and

$$
\liminf _{n \rightarrow \infty} P^{n} \mu_{0}\left(A_{i}\right)>0 \quad \text { for } i=1, \ldots, k .
$$

Proof. Fix $\varepsilon>0$. Set

$$
k=\min \left\{m \in \mathbb{N}: \exists \sigma \in(0, \varepsilon) \mathcal{T}^{m}(\sigma) \neq \emptyset\right\} .
$$

Choose $\eta \in(0, \varepsilon)$ such that $\mathcal{T}^{k}(\eta) \neq \emptyset$. Choose $C=\bigcup_{i=1}^{k} \widetilde{A}_{i}$ where $\widetilde{A}_{i}$ are closed balls with radius $\eta$ and $\alpha>0$ such that

$$
\liminf _{n \rightarrow \infty} P^{n} \mu(C)>\alpha \quad \text { for } \mu \in \mathcal{M}_{1} .
$$

Choose $\gamma>0$ such that

$$
\eta+\gamma<\varepsilon \quad \text { and } \quad k \gamma<\alpha .
$$

Set $\widetilde{\varepsilon}=\gamma^{2}$. Let $\widetilde{\alpha}>0, p \in \mathbb{N}$ and $\widetilde{C}=\bigcup_{i=1}^{p} D_{i}$, where $D_{i}$ are closed balls with radius $\widetilde{\varepsilon}$, be chosen according to the semi-concentrating property of $P$ for $\widetilde{\varepsilon}$. For every $\mu \in \mathcal{M}_{1}$ we define $\mathcal{L}(\mu)$ to be the set of all $j \in\{1, \ldots, p\}$ such that there exists $n \in \mathbb{N}$ satisfying

$$
P^{n} \mu\left(D_{j}\right) \geq \widetilde{\alpha} / p \text {. }
$$

Obviously $\mathcal{L}(\mu) \neq \emptyset$. Further, it follows easily that $j \in \mathcal{L}(\mu)$ iff there exists $n \in \mathbb{N}$ such that

$$
P^{n} \mu \geq(\widetilde{\alpha} / p) \nu \quad \text { for some } \nu \in \mathcal{M}_{1}^{D_{j}} .
$$


We proceed to show that for every $i \in\{1, \ldots, k\}$ there exists $\mu \in \mathcal{M}_{1}$ such that for every $j \in \mathcal{L}(\mu)$ and $x \in D_{j}$ we have

$$
P^{n} \delta_{x}\left(\widetilde{A}_{i}\right) \geq \alpha / k \quad \text { for some } n \in \mathbb{N} .
$$

We can assume that $i=1$. Suppose, contrary to our claim, that for every $\mu \in \mathcal{M}_{1}$ there exist $j \in \mathcal{L}(\mu)$ and $x \in D_{j}$ such that $P^{n} \delta_{x}\left(\widetilde{A}_{1}\right)<\alpha / k$ for $n \in \mathbb{N}$. From $(2.1)$ we conclude that $P^{n} \delta_{x}\left(\widetilde{A}_{i_{n}}\right) \geq \alpha / k$ for all sufficiently large $n \in \mathbb{N}$, where $i_{n} \in\{1, \ldots, k\}$ and $i_{n} \neq 1$. Since $\left\|\delta_{x}-\nu\right\| \leq \operatorname{diam} D_{j} \leq \gamma^{2}$ for $\nu \in \mathcal{M}_{1}^{D_{j}}$ and $P$ is nonexpansive, Lemma 2.1 shows that

$$
P^{n} \nu\left(\mathcal{N}^{0}\left(\widetilde{A}_{i_{n}}, \gamma\right)\right) \geq \alpha / k-\gamma
$$

for $\nu \in \mathcal{M}_{1}^{D_{j}}$ and all sufficiently large $n \in \mathbb{N}$. Since $i_{n} \neq 1$, we then obtain

$$
P^{n} \nu\left(\bigcup_{i=2}^{k} \mathcal{N}^{0}\left(\widetilde{A}_{i}, \gamma\right)\right) \geq \alpha / k-\gamma \quad \text { for } \nu \in \mathcal{M}_{1}^{D_{j}}
$$

From (2.3) we conclude that $P^{n_{0}} \mu \geq(\widetilde{\alpha} / p) \nu$ for some $\nu \in \mathcal{M}_{1}^{D_{j}}, j \in \mathcal{L}(\mu)$, and $n_{0} \in \mathbb{N}$. Consequently,

$$
P^{n_{0}+n} \mu \geq(\widetilde{\alpha} / p) P^{n} \nu \quad \text { for } n \in \mathbb{N}
$$

and so

$$
P^{n_{0}+n} \mu\left(\bigcup_{i=2}^{k} \mathcal{N}^{0}\left(\widetilde{A}_{i}, \gamma\right)\right) \geq(\widetilde{\alpha} / p)(\alpha / k-\gamma)
$$

for all sufficiently large $n \in \mathbb{N}$. Thus

$$
\liminf _{n \rightarrow \infty} P^{n} \mu\left(\bigcup_{i=2}^{k} \mathcal{N}^{0}\left(\widetilde{A}_{i}, \gamma\right)\right) \geq(\widetilde{\alpha} / p)(\alpha / k-\gamma) \quad \text { for } \mu \in \mathcal{M}_{1} .
$$

From (2.2) we conclude that $\bigcup_{i=2}^{k} \mathcal{N}^{0}\left(\widetilde{A}_{i}, \gamma\right) \in \mathcal{C}^{k-1}(\eta+\gamma)$, hence $\mathcal{T}^{k-1}(\eta+\gamma)$ $\neq \emptyset$, contrary to the definition of $k$.

Let $\mu_{i} \in \mathcal{M}_{1}, 1 \leq i \leq k$, be such that for every $j \in \mathcal{L}\left(\mu_{i}\right)$ and $x \in D_{j}$ condition (2.4) holds. Fix $i \in\{1, \ldots, k\}$. For every $j \in \mathcal{L}\left(\mu_{i}\right)$ choose $x_{j} \in D_{j}$ and an integer $n_{j}$ such that

$$
P^{n_{j}} \delta_{x_{j}}\left(\widetilde{A}_{i}\right) \geq \alpha / k
$$

By Lemma 2.1 we obtain

$$
P^{n_{j}} \nu\left(\mathcal{N}\left(\widetilde{A}_{i}, \gamma\right)\right) \geq \alpha / k-\gamma \quad \text { for } \nu \in \mathcal{M}_{1}^{D_{j}} .
$$

Set $N_{i}=\max _{j \in \mathcal{L}\left(\mu_{i}\right)} n_{j}$ and $A_{i}=\mathcal{N}\left(\widetilde{A}_{i}, \gamma\right)$. From (2.1) and (2.2) it follows that $\bigcup_{i=1}^{k} A_{i} \in \mathcal{T}^{k}(\varepsilon)$. Define $\bar{\mu}_{i} \in \mathcal{M}_{1}$ by the formula

$$
\bar{\mu}_{i}=\frac{\mu_{i}+P \mu_{i}+\ldots+P^{N_{i}} \mu_{i}}{N_{i}+1} .
$$


It is easy to check that

$$
\liminf _{n \rightarrow \infty} P^{n} \bar{\mu}_{i}\left(A_{i}\right) \geq \widetilde{\alpha}(\alpha / k-\gamma) /\left(N_{i}+1\right)>0
$$

for $i \in\{1, \ldots, k\}$. Write

$$
\mu_{0}=\frac{\bar{\mu}_{1}+\ldots+\bar{\mu}_{k}}{k} .
$$

From (2.5) and the linearity of $P$ we have

$$
\liminf _{n \rightarrow \infty} P^{n} \mu_{0}\left(A_{i}\right)>0 \quad \text { for } i=1, \ldots, k .
$$

Lemma 2.4. Let $P$ be a nonexpansive Markov operator, $A \in \mathcal{B}(X)$ and $\varepsilon>0$. Assume that $\operatorname{diam} A \leq \varepsilon^{2} / 16$ and there exists $\mu \in \mathcal{M}_{1}$ such that

$$
\liminf _{n \rightarrow \infty} P^{n} \mu(A)>0 \text {. }
$$

Then there exists $C \in \mathcal{C}(\varepsilon)$ such that $P^{n} \nu(C)>1-\varepsilon$ for $n \in \mathbb{N}$ and $\nu \in \mathcal{M}_{1}^{A}$.

Proof. Choose $\alpha>0$ such that

$$
\liminf _{n \rightarrow \infty} P^{n} \mu(A) \geq \alpha \text {. }
$$

If $P^{n} \mu(A) \geq \alpha / 2$, then

$$
P^{n} \mu \geq(\alpha / 2) \nu_{n}
$$

for some $\nu_{n} \in \mathcal{M}_{1}^{A}$. Define

$$
\delta=\sup \left\{\gamma \geq 0: \exists C_{\varepsilon / 2} \in \mathcal{C}(\varepsilon / 2) \liminf _{n \rightarrow \infty} P^{n} \mu\left(C_{\varepsilon / 2}\right) \geq \gamma\right\} .
$$

Choose $\gamma \geq 0$ and $C_{\varepsilon / 2} \in \mathcal{C}(\varepsilon / 2)$ such that $0 \leq \delta-\gamma<\alpha \varepsilon / 8$ and

$$
\liminf _{n \rightarrow \infty} P^{n} \mu\left(C_{\varepsilon / 2}\right) \geq \gamma
$$

We are now in a position to show that

$$
P^{n} \nu\left(\mathcal{N}^{0}\left(C_{\varepsilon / 2}, \varepsilon / 2\right)\right) \geq 1-\varepsilon / 2 \quad \text { for } n \in \mathbb{N} \text { and } \nu \in \mathcal{M}_{1}^{A} .
$$

On the contrary, suppose that for some $\nu_{0} \in \mathcal{M}_{1}^{A}$ and $n_{0} \in \mathbb{N}$,

$$
P^{n_{0}} \nu_{0}\left(\mathcal{N}^{0}\left(C_{\varepsilon / 2}, \varepsilon / 2\right)\right)<1-\varepsilon / 2 .
$$

By the Ulam theorem, this implies that there exists a compact set $K \subset$ $X \backslash \mathcal{N}^{0}\left(C_{\varepsilon / 2}, \varepsilon / 2\right)$ such that $P^{n_{0}} \nu_{0}(K) \geq \varepsilon / 2$. Since $P$ is nonexpansive, we have

$$
\left\|P^{n_{0}} \nu_{0}-P^{n_{0}} \nu\right\| \leq\left\|\nu_{0}-\nu\right\| \leq \operatorname{diam} A \leq \varepsilon^{2} / 16
$$

for $\nu \in \mathcal{M}_{1}^{A}$. Lemma 2.1 now shows that $P^{n_{0}} \nu\left(\mathcal{N}^{0}(K, \varepsilon / 4)\right) \geq \varepsilon / 4$. Put $B=\mathcal{N}(K, \varepsilon / 4)$. Obviously $B \in \mathcal{C}(\varepsilon / 2)$ and consequently $B \cup C_{\varepsilon / 2} \in \mathcal{C}(\varepsilon / 2)$. Applying (2.6) we see that

$$
P^{n+n_{0}} \mu(B) \geq \frac{\alpha}{2} P^{n_{0}} \nu_{n}(B) \geq \frac{\alpha \varepsilon}{8}
$$


for all sufficiently large $n$. Since $B \cap C_{\varepsilon / 2}=\emptyset$, we see that

$$
\begin{aligned}
\liminf _{n \rightarrow \infty} P^{n} \mu\left(B \cup C_{\varepsilon / 2}\right) & \geq \liminf _{n \rightarrow \infty} P^{n} \mu(B)+\liminf _{n \rightarrow \infty} P^{n} \mu\left(C_{\varepsilon / 2}\right) \\
& \geq \alpha \varepsilon / 8+\gamma>\delta,
\end{aligned}
$$

which contradicts the definition of $\delta$. Thus (2.8) holds. Put $C=\mathcal{N}\left(C_{\varepsilon / 2}, \varepsilon / 2\right)$ and note that $C \in \mathcal{C}(\varepsilon)$.

Lemma 2.5. Let $P$ be a nonexpansive semi-concentrating Markov operator. Then for every $\varepsilon>0$ there exists $C \in \mathcal{C}(\varepsilon)$ satisfying

$$
\liminf _{n \rightarrow \infty} P^{n} \mu(C) \geq 1-\varepsilon \quad \text { for } \mu \in \mathcal{M}_{1} .
$$

Proof. Fix $\varepsilon>0$. By Lemma 2.3 there exist an integer $k$, a sequence $\left(A_{1}, \ldots, A_{k}\right)$ with $A_{i} \in \mathcal{B}(X)$ and $\operatorname{diam} A_{i} \leq \varepsilon^{2} / 16$ for $i=1, \ldots, k$, and a measure $\mu_{0} \in \mathcal{M}_{1}$ such that $\bigcup_{i=1}^{k} A_{i} \in \mathcal{T}^{k}\left(\varepsilon^{2} / 16\right)$ and

$$
\liminf _{n \rightarrow \infty} P^{n} \mu_{0}\left(A_{i}\right)>0 \quad \text { for } i=1, \ldots, k .
$$

Lemma 2.4 now shows that there exists a sequence $\left(C_{1}, \ldots, C_{k}\right)$ with $C_{i} \in$ $\mathcal{C}(\varepsilon / 2)$ for $i=1, \ldots, k$ satisfying $P^{n} \nu\left(C_{i}\right)>1-\varepsilon / 2$ for $n \in \mathbb{N}, \nu \in \mathcal{M}_{1}^{A_{i}}$ and $i=1, \ldots, k$. Set $C=\bigcup_{i=1}^{k} C^{i}$ and observe that $C \in \mathcal{C}(\varepsilon)$. Moreover,

$$
P^{n} \nu(C)>1-\varepsilon / 2 \quad \text { for } n \in \mathbb{N} \text { and } \nu \in \bigcup_{i=1}^{k} \mathcal{M}_{1}^{A_{i}} \text {. }
$$

Since $\bigcup_{i=1}^{k} A_{i} \in \mathcal{T}^{k}\left(\varepsilon^{2} / 16\right)$, there exists $\widetilde{\alpha}>0$ such that

$$
\liminf _{n \rightarrow \infty} P^{n} \mu\left(\bigcup_{i=1}^{k} A_{i}\right)>\widetilde{\alpha} \quad \text { for } \mu \in \mathcal{M}_{1} .
$$

Set $\alpha=\widetilde{\alpha} / k$ and define

$$
\eta=\sup \left\{\gamma \geq 0: \liminf _{n \rightarrow \infty} P^{n} \mu(C) \geq \gamma \text { for } \mu \in \mathcal{M}_{1}\right\} .
$$

It remains to prove that $\eta \geq 1-\varepsilon / 2$. Suppose, contrary to our claim, that $\eta<1-\varepsilon / 2$. Hence

$$
\eta>\frac{\eta}{1-\alpha}-\frac{\alpha}{1-\alpha}(1-\varepsilon / 2)
$$

Choose a real number $\gamma$ such that

$$
\eta>\gamma>\frac{\eta}{1-\alpha}-\frac{\alpha}{1-\alpha}(1-\varepsilon / 2) .
$$

Then

$$
\liminf _{n \rightarrow \infty} P^{n} \mu(C) \geq \gamma \quad \text { for } \mu \in \mathcal{M}_{1} .
$$


Fix $\mu \in \mathcal{M}_{1}$. From (2.11) it follows that there exist $n_{0} \in \mathbb{N}$ and $i \in\{1, \ldots, k\}$ such that $P^{n_{0}} \mu\left(A_{i}\right)>\alpha$. Define

$$
\begin{aligned}
\nu(B) & =\frac{P^{n_{0}} \mu\left(B \cap A_{i}\right)}{P^{n_{0}} \mu\left(A_{i}\right)}, \\
\widetilde{\mu}(B) & =\frac{1}{1-\alpha}\left\{P^{n_{0}} \mu(B)-\alpha \nu(B)\right\} \quad \text { for } B \in \mathcal{B}(X) .
\end{aligned}
$$

Then $P^{n_{0}} \mu=(1-\alpha) \widetilde{\mu}+\alpha \nu$. Obviously $\nu \in \bigcup_{i=1}^{k} \mathcal{M}_{1}^{A_{i}}$. By (2.10), (2.12) and the linearity of $P$ we now obtain

$$
\begin{aligned}
\liminf _{n \rightarrow \infty} P^{n_{0}+n} \mu(C) & \geq(1-\alpha) \liminf _{n \rightarrow \infty} P^{n} \widetilde{\mu}(C)+\alpha \liminf _{n \rightarrow \infty} P^{n} \nu(C) \\
& >(1-\alpha) \gamma+\alpha(1-\varepsilon / 2)>\eta .
\end{aligned}
$$

Since $\mu \in \mathcal{M}_{1}$ is arbitrary, we see that

$$
\liminf _{n \rightarrow \infty} P^{n} \mu(C) \geq(1-\alpha) \gamma+\alpha(1-\varepsilon / 2)>\eta \quad \text { for all } \mu \in \mathcal{M}_{1},
$$

which contradicts the definition of $\eta$.

Combining Lemmas 2.2 and 2.5 we obtain the following theorem:

Theorem 2.1. Let $P: \mathcal{M} \rightarrow \mathcal{M}$ be a nonexpansive semi-concentrating Markov operator. Then $P$ has an invariant distribution.

Theorem 2.2. Let $P: \mathcal{M} \rightarrow \mathcal{M}$ be a nonexpansive semi-concentrating Markov operator. Then

(i) $\omega(\mu) \neq \emptyset$ for every $\mu \in \mathcal{M}_{1}$,

(ii) $\Omega=\bigcup_{\mu \in \mathcal{M}_{1}} \omega(\mu)$ is tight.

Proof. (i) Fix $\varepsilon>0$ and $\mu \in \mathcal{M}_{1}$. By Lemma 2.5 there exists a set $C \in \mathcal{C}(\varepsilon)$ such that

$$
\liminf _{n \rightarrow \infty} P^{n} \mu(C)>1-\varepsilon
$$

By the Ulam theorem we can find a compact set $K \subset X$ such that

$$
P^{n} \mu(K \cup C)>1-\varepsilon \quad \text { for } n \in \mathbb{N} .
$$

Since $K \cup C \in \mathcal{C}(\varepsilon)$, Lemma 2.2 shows that the sequence $\left(P^{n} \mu\right)_{n \geq 1}$ is tight. From this and the Prokhorov theorem (see [3]) it follows that $\omega(\mu) \neq \emptyset$.

(ii) To prove the tightness of $\Omega$ fix $\varepsilon>0$. Again, by Lemma 2.5 there exists a sequence $\left(C_{i}\right)_{i \geq 1}$ of subsets of $X$ such that

$$
C_{i} \in \mathcal{C}\left(\varepsilon / 2^{i}\right) \quad \text { and } \quad \liminf _{n \rightarrow \infty} P^{n} \mu\left(C_{i}\right) \geq 1-\varepsilon / 2^{i} \quad \text { for } i \in \mathbb{N} .
$$

Define $K=\bigcap_{i=1}^{\infty} \mathcal{N}\left(C_{i}, \varepsilon / 2^{i}\right)$ and observe that $K$ is compact. We are going to show that $\widetilde{\mu}(K) \geq 1-\varepsilon$ for $\widetilde{\mu} \in \Omega$. Fix $\widetilde{\mu} \in \Omega$ and let $\mu \in \mathcal{M}_{1}$ be such that $\widetilde{\mu} \in \omega(\mu)$. Let $\left(n_{m}\right)_{m \geq 1}$ be a sequence of integers such that $\left\|P^{n_{m}} \mu-\widetilde{\mu}\right\| \rightarrow 0$ 
as $m \rightarrow \infty$. Then by the Aleksandrov theorem we have

$$
\begin{aligned}
\widetilde{\mu}\left(\mathcal{N}\left(C_{i}, \varepsilon / 2^{i}\right)\right) & \geq \widetilde{\mu}\left(\mathcal{N}^{0}\left(C_{i}, \varepsilon / 2^{i}\right)\right) \geq \limsup _{m \rightarrow \infty} P^{n_{m}} \mu\left(\mathcal{N}^{0}\left(C_{i}, \varepsilon / 2^{i}\right)\right) \\
& \geq \limsup _{m \rightarrow \infty} P^{n_{m}} \mu\left(C_{i}\right) \geq 1-\varepsilon / 2^{i} \quad \text { for } i \in \mathbb{N} .
\end{aligned}
$$

Hence

$$
\widetilde{\mu}(X \backslash K) \leq \sum_{i=1}^{\infty} \widetilde{\mu}\left(X \backslash \mathcal{N}\left(C_{i}, \varepsilon / 2^{i}\right)\right)<\sum_{i=1}^{\infty} \varepsilon / 2^{i}=\varepsilon,
$$

which finishes the proof of (ii).

3. Iterated function systems. Assume we are given a family of continuous transformations $S_{i}: X \rightarrow X, i=1, \ldots, N$, and a probabilistic vector

$$
\left(p_{1}(x), \ldots, p_{N}(x)\right), \quad p_{i}(x) \geq 0, \quad \sum_{i=1}^{N} p_{i}(x)=1 .
$$

The pair of sequences $(S, p)_{N}=\left(S_{1}, \ldots, S_{N} ; p_{1}, \ldots, p_{N}\right)$ is called an iterated function system.

We consider some special Markov operators describing the evolution of measures due to the action of randomly chosen transformations. A simple but somewhat inexact description of this process goes as follows. Choose $x_{0} \in X$. When an initial point $x_{0}$ is chosen, we randomly select an integer from $\{1, \ldots, N\}$ in such a way that the probability of choosing $k$ is $p_{k}\left(x_{0}\right)$, $k=1, \ldots, N$. When a number $k_{0}$ is drawn we define $x_{1}=S_{k_{0}}\left(x_{0}\right)$. Having $x_{1}$ we select $k_{1}$ according to the distribution $p_{1}\left(x_{1}\right), \ldots, p_{N}\left(x_{1}\right)$, we define $x_{2}=S_{k_{1}}\left(x_{1}\right)$ and so on. Denoting by $\mu_{n}, n=0,1, \ldots$, the distribution of $x_{n}$, i.e. $\mu_{n}(A)=\operatorname{Prob}\left(x_{n} \in A\right)$, we define $P$ as the transition operator such that $\mu_{n+1}=P \mu_{n}$. We can prove (see $[2,7,12]$ ) that it must be of the form

$$
P \mu(A)=\sum_{i=1}^{N} \int_{S_{i}^{-1}(A)} p_{i} d \mu .
$$

Moreover, it is a Feller operator and its adjoint operator is given by

$$
U f(x)=\sum_{i=1}^{N} p_{i}(x) f\left(S_{i}(x)\right) .
$$

Now assume that

$$
\sum_{i=1}^{N}\left|p_{i}(x)-p_{i}(y)\right| \leq \omega(\varrho(x, y)) \quad \text { for } x, y \in X,
$$

where $\omega: \mathbb{R}_{+} \rightarrow \mathbb{R}_{+}$is a continuous function. The function $\omega$ is called a modulus of continuity. Further, we assume that $\omega$ satisfies the Dini condition, 
i.e. $\omega: \mathbb{R}_{+} \rightarrow \mathbb{R}_{+}$is a nondecreasing concave function such that

$$
\int_{0}^{a} \frac{\omega(t)}{t} d t<\infty \quad \text { for some } a>0 .
$$

We do not require the $S_{i}$ 's to be contractions, but require only an average contractivity condition between points, as in [2]:

$$
\sum_{i=1}^{N} p_{i}(x) \varrho\left(S_{i}(x), S_{i}(y)\right) \leq r \varrho(x, y) \quad \text { for } x, y \in X,
$$

where $r<1$.

Let us start with the following observation. The asymptotic stability of a Markov operator $P$ acting on measures defined on a metric space $(X, \varrho)$ may be verified without the precise knowledge of the metric $\varrho$. What is important is just the space $C(X)$ of all bounded continuous functions. We may change the metric $\varrho$ in such a way that $P$ becomes nonexpansive and the family of all bounded continuous functions remains the same. Conditions (3.3) and (3.4) allow us to do it. Summarizing, we have the following:

Proposition 3.1. Let an iterated function system $(S, p)_{N}$ satisfy condition (3.4). If the modulus of continuity $\omega$ defined by (3.3) satisfies the Dini condition, then there exists a new metric $\bar{\varrho}$ such that the new space $(X, \bar{\varrho})$ remains a Polish space and the Markov operator $P$ given by (3.1) is nonexpansive. Moreover, bounded sets in both spaces remain the same and for every $\varepsilon>0$ there exists $\eta>0$ such that $\operatorname{diam}_{\bar{\varrho}}(C)<\varepsilon$ if $\operatorname{diam}_{\varrho}(C)<\eta$ for $C \subset X$.

The above theorem (see [12, Proposition 6.1]) was formulated in the case when $(X, \varrho)$ is a locally compact, $\sigma$-compact metric space but its proof remains valid for every Polish space.

Moreover, if we want to verify the global concentrating property or the semi-concentrating property for $P$, the last theorem allows us to verify it in the former metric.

TheOREM 3.1. Let an iterated function system $(S, p)_{N}=\left(S_{1}, \ldots, S_{N}\right.$; $\left.p_{1}, \ldots, p_{n}\right)$ satisfy condition (3.4). Assume that the modulus of continuity $\omega$ defined by (3.3) satisfies the Dini condition. If there exists $\delta>0$ such that

$$
\sum_{i} p_{i}(x) p_{i}(y) \geq \delta \quad \text { for } x, y \in X,
$$

where the summation is taken over all integers $i, 1 \leq i \leq N$, such that $\varrho\left(S_{i}(x), S_{i}(y)\right) \leq r \varrho(x, y)$, then the Markov operator $P$ given by (3.1) is semi-concentrating and consequently has an invariant distribution.

Proof. Since the assumptions of Proposition 3.1 are satisfied, we may assume that $P$ is nonexpansive. Further, Theorem 4.2 in [18] shows that $P$ 
is globally concentrating. Hence there exists a set $Y \in \mathcal{B}_{\mathrm{b}}(X)$ such that

$$
\liminf _{n \rightarrow \infty} P^{n} \mu(Y)>1 / 2 \quad \text { for } \mu \in \mathcal{M}_{1} \text {. }
$$

To show that $P$ is semi-concentrating fix $\varepsilon>0$. Choose an integer $m$ such that

$$
r^{m} \operatorname{diam} Y<\varepsilon \text {. }
$$

Fix $x \in X$ and define

$$
C=\bigcup_{i_{1}, \ldots, i_{m}=1}^{N} B\left(S_{i_{m}} \circ \ldots \circ S_{i_{1}}(x), \varepsilon\right) .
$$

Let $\delta>0$ be such that (3.5) is satisfied and set $\bar{\delta}=\delta / N$. For every sequence $\left(i_{1}, \ldots, i_{m}\right) \in\{1, \ldots, N\}^{m}$ denote by $Y_{\left(i_{1}, \ldots, i_{m}\right)}$ the set of all $y \in Y$ such that

$$
S_{i_{m}} \circ \ldots \circ S_{i_{1}}(y) \in B\left(S_{i_{m}} \circ \ldots \circ S_{i_{1}}(x), r^{m} \varrho(x, y)\right)
$$

and

$$
p_{i_{1}}(y) \cdot \ldots \cdot p_{i_{m}}\left(S_{i_{m-1}} \circ \ldots \circ S_{i_{1}}(y)\right) \geq \bar{\delta}^{m} .
$$

From (3.4) and (3.5) it follows that

$$
Y=\bigcup_{i_{1}, \ldots, i_{m}=1}^{N} Y_{\left(i_{1}, \ldots, i_{m}\right)} .
$$

In fact, by an induction argument for every $y \in Y$ there is a sequence $\left(k_{1}, \ldots, k_{m}\right) \in\{1, \ldots, N\}^{m}$ such that

$$
\varrho\left(S_{k_{m}} \circ \ldots \circ S_{k_{1}}(x), S_{k_{m}} \circ \ldots \circ S_{k_{1}}(y)\right) \leq r^{m} \varrho(x, y)
$$

and

$$
p_{k_{1}}(y) \cdot \ldots \cdot p_{k_{m}}\left(S_{k_{m-1}} \circ \ldots \circ S_{k_{1}}(y)\right) \geq \bar{\delta}^{m} .
$$

Consequently, $y \in Y_{\left(k_{1}, \ldots, k_{m}\right)} \subset \bigcup_{i_{1}, \ldots, i_{m}=1}^{N} Y_{\left(i_{1}, \ldots, i_{m}\right)}$.

We are going to show that $P$ satisfies condition (1.11) with $\alpha=$ $\bar{\delta}^{m} /\left(2 N^{m}\right)$. Fix $\mu \in \mathcal{M}_{1}$. According to (3.6), we may choose an integer $n_{0}$ such that

$$
P^{n} \mu(Y) \geq 1 / 2 \quad \text { for } n \geq n_{0} .
$$

From this and condition (3.9) it follows that

$$
P^{n} \mu\left(Y_{\left(k_{1}^{n}, \ldots, k_{m}^{n}\right)}\right) \geq 1 /\left(2 N^{m}\right)
$$

for $n \geq n_{0}$ and some $\left(k_{1}^{n}, \ldots, k_{m}^{n}\right) \in\{1, \ldots, N\}^{m}$.

By an induction argument for $n \in \mathbb{N}$ we have

$$
\begin{aligned}
P^{n+m} \mu(C)= & \left\langle\mathbf{1}_{C}, P^{n+m} \mu\right\rangle=\left\langle U^{m} \mathbf{1}_{C}, P^{n} \mu\right\rangle \\
= & \sum_{i_{1}, \ldots, i_{m}=1}^{N} \int_{X} p_{i_{1}}(y) \cdot \ldots \cdot p_{i_{m}}\left(S_{i_{m-1}} \circ \ldots \circ S_{i_{1}}(y)\right) \\
& \times \mathbf{1}_{C}\left(S_{i_{m}} \circ \ldots \circ S_{i_{1}}(y)\right) P^{n} \mu(d y) .
\end{aligned}
$$


Therefore

$$
P^{n+m} \mu(C) \geq \bar{\delta}^{m} /\left(2 N^{m}\right)=\alpha \quad \text { for } n \geq n_{0} .
$$

Consequently,

$$
\liminf _{n \rightarrow \infty} P^{n} \mu(C) \geq \alpha \quad \text { for all } \mu \in \mathcal{M}_{1} .
$$

Since $\varepsilon>0$ is arbitrary, this completes the proof of the semi-concentrating property. An application of Theorem 2.1 finishes the proof.

TheOREM 3.2. Under the assumptions of Theorem 3.1, the Markov operator $P$ is asymptotically stable.

Proof. By Theorem 3.1, $P$ has an invariant distribution $\mu_{*}$. It remains to verify (1.9). When an invariant distribution exists this condition is equivalent to a more symmetric relation

$$
\lim _{n \rightarrow \infty}\left\|P^{n}\left(\mu_{1}-\mu_{2}\right)\right\|=0 \quad \text { for } \mu_{1}, \mu_{2} \in \mathcal{M}_{1} .
$$

By Proposition 3.1, we may assume that $P$ is nonexpansive. Analysis similar to that in the proof of Theorem 8.1 in [12] shows that to verify (3.10) it is enough to prove that for every $\varepsilon>0$ there is a number $\alpha>0$ with the following property: for every $\mu_{1}, \mu_{2} \in \mathcal{M}_{1}$ there exist a Borel set $A$ with $\operatorname{diam} A \leq \varepsilon$ and an integer $n_{0}$ such that

$$
P^{n_{0}} \mu_{i}(A) \geq \alpha \quad \text { for } i=1,2 .
$$

Fix $\varepsilon>0$. According to Theorem 2.2(ii) there is a compact set $K$ such that

$$
\bar{\mu}(K) \geq 4 / 5 \quad \text { for } \bar{\mu} \in \Omega=\bigcup_{\mu \in \mathcal{M}_{1}} \omega(\mu) .
$$

Choose an integer $m$ such that

$$
r^{m} \operatorname{diam} K \leq \varepsilon / 3
$$

and for every $x \in K$ and $\left(j_{1}, \ldots, j_{m}\right) \in\{1, \ldots, N\}^{m}$ define

$$
\Pi_{\left(j_{1}, \ldots, j_{m}\right)}(x)=p_{j_{1}}(x) \cdot \ldots \cdot p_{j_{m}}\left(S_{j_{m-1}} \circ \ldots \circ S_{j_{1}}(x)\right) .
$$

For every $x \in K$ and $\left(j_{1}, \ldots, j_{m}\right) \in\{1, \ldots, N\}^{m}$ define the open neighbourhood $O_{\left(j_{1}, \ldots, j_{m}\right)}(x)$ of $x$ in the following way:

1) if $\Pi_{\left(j_{1}, \ldots, j_{m}\right)}(x)>0$, then

$$
\begin{array}{r}
O_{\left(j_{1}, \ldots, j_{m}\right)}(x)=\left\{y \in X: \varrho\left(S_{j_{m}, \ldots, j_{1}}(x), S_{j_{m}, \ldots, j_{1}}(y)\right)<\varepsilon / 3,\right. \\
\left.\Pi_{\left(j_{1}, \ldots, j_{m}\right)}(y)>\Pi_{\left(j_{1}, \ldots, j_{m}\right)}(x) / 2\right\},
\end{array}
$$

2) if $\Pi_{\left(j_{1}, \ldots, j_{m}\right)}(x)=0$, then

$$
O_{\left(j_{1}, \ldots, j_{m}\right)}(x)=\left\{y \in X: \varrho\left(S_{j_{m}, \ldots, j_{1}}(x), S_{j_{m}, \ldots, j_{1}}(y)\right)<\varepsilon / 3\right\},
$$

where $S_{j_{m}, \ldots, j_{1}}(x)=S_{j_{m}} \circ \ldots \circ S_{j_{1}}(x)$. 
Set

$$
O_{x}=\bigcap_{j_{1}, \ldots, j_{m}=1}^{N} O_{\left(j_{1}, \ldots, j_{m}\right)}(x) \quad \text { for } x \in X .
$$

Since $K$ is a compact set there is a finite covering

$$
K \subset \bigcup_{i=1}^{q} O_{x_{i}}
$$

Set $G=\bigcup_{i=1}^{q} O_{x_{i}}$. Let $\delta>0$ be such that (3.5) is satisfied and set $\bar{\delta}=\delta / N$. We are going to show that $P$ satisfies (3.11) with $\alpha=\bar{\delta}^{m} /(4 q)$. In fact, let $\mu_{1}, \mu_{2} \in \mathcal{M}_{1}$ be given. Set $\mu_{0}=\left(\mu_{1}+\mu_{2}\right) / 2$. According to Theorems 3.1 and 2.2(i) there exists $\bar{\mu} \in \mathcal{M}_{1}$ such that $\bar{\mu} \in \omega\left(\mu_{0}\right)$. Consequently, there exists a sequence $\left(m_{n}\right)_{n \geq 1}$ such that

$$
\lim _{n \rightarrow \infty}\left\|P^{m_{n}} \mu_{0}-\bar{\mu}\right\|=0
$$

Since (3.16) is equivalent to the weak convergence of $\left(P^{m_{n}} \mu_{0}\right)_{n \geq 1}$ to $\bar{\mu}$ and $G$ is open, the Aleksandrov theorem implies

$$
\liminf _{n \rightarrow \infty} P^{m_{n}} \mu_{0}(G) \geq \bar{\mu}(G) .
$$

From this and (3.12), (3.15) it follows that there exists an integer $n$ such that

$$
P^{n} \mu_{0}(G)=\left(P^{n} \mu_{1}(G)+P^{n} \mu_{2}(G)\right) / 2 \geq 3 / 4 .
$$

Hence $P^{n} \mu_{k}(G) \geq 1 / 2$ for $k=1,2$. Then (3.15) implies that there exist $s, t \in\{1, \ldots, q\}$ such that

$$
P^{n} \mu_{1}\left(O_{x_{s}}\right) \geq 1 /(2 q) \text { and } P^{n} \mu_{2}\left(O_{x_{t}}\right) \geq 1 /(2 q) .
$$

Write for simplicity $O_{1}=O_{x_{s}}$ and $O_{2}=O_{x_{t}}$. From (3.4) we conclude that there is $\left(i_{1}, \ldots, i_{m}\right) \in\{1, \ldots, N\}^{m}$ such that

$$
\varrho\left(S_{i_{m}} \circ \ldots \circ S_{i_{1}}\left(x_{s}\right), S_{i_{m}} \circ \ldots \circ S_{i_{1}}\left(x_{t}\right)\right) \leq r^{m} \varrho\left(x_{s}, x_{t}\right) \leq \varepsilon / 3
$$

and

$$
\Pi_{\left(i_{1}, \ldots, i_{m}\right)}\left(x_{s}\right) \geq \bar{\delta}^{m}, \quad \Pi_{\left(i_{1}, \ldots, i_{m}\right)}\left(x_{t}\right) \geq \bar{\delta}^{m} .
$$

Define

$$
A=A_{1} \cup A_{2} \quad \text { where } \quad A_{k}=S_{i_{m}} \circ \ldots \circ S_{i_{1}}\left(O_{k}\right), k=1,2 .
$$

According to (3.13), (3.14) and (3.17) we have $\operatorname{diam} A \leq \varepsilon$ and $\Pi_{\left(i_{1}, \ldots, i_{m}\right)}(y)$ 
$\geq \bar{\delta}^{m} / 2$ for $y \in O_{1} \cup O_{2}$. On the other hand, from (3.17) we obtain

$$
\begin{aligned}
P^{m+n} \mu_{k}(A) & =\left\langle U^{m+n} \mathbf{1}_{A}, \mu_{k}\right\rangle=\left\langle U^{m} \mathbf{1}_{A}, P^{n} \mu_{k}\right\rangle \\
& \geq \sum_{j_{1}, \ldots, j_{m}=1}^{N} \int_{X} \Pi_{\left(j_{1}, \ldots, j_{m}\right)}(y) \mathbf{1}_{A_{k}}\left(S_{j_{m}} \circ \ldots \circ S_{j_{1}}(y)\right) P^{n} \mu_{k}(d y) \\
& \geq \bar{\delta}^{m} P^{n} \mu_{k}\left(O_{k}\right) / 2=\bar{\delta}^{m} /(4 q) \quad \text { for } k=1,2,
\end{aligned}
$$

which finishes the proof.

Acknowledgements. This research was supported by the Foundation for Polish Science and the State Committee for Scientific Research (Poland) Grant No. 2 P03A 010 16. It was also supported by a Marie Curie Fellowship of the European Community programme "Improving the Human Research Potential and the Socio-Economic Knowledge Base" under contract number HPMF-CT-2000-00824.

\section{References}

[1] M. F. Barnsley and S. Demko, Iterated function systems and the global construction of fractals, Proc. Roy. Soc. London Sect. A 399 (1985), 243-275.

[2] M. F. Barnsley, S. G. Demko, J. H. Elton and J. S. Geronimo, Invariant measures arising from iterated function systems with place dependent probabilities, Ann. Inst. H. Poincaré 24 (1988), 367-394.

[3] P. Billingsley, Convergence of Probability Measures, Wiley, 1968.

[4] P. M. Centore and E. R. Vrscay, Continuity of attractors and invariant measures for iterated function systems, Canad. Math. Bull. 37 (1994), 315-329.

[5] E. Çinlar, Introduction to Stochastic Processes, Prentice-Hall, 1975.

[6] R. M. Dudley, Probabilities and Metrics, Aarhus Universitet, 1976.

[7] J. H. Elton and M. Piccioni, Iterated function systems arising from recursive estimation problems, Probab. Theory Related Fields 91 (1992), 103-114.

[8] R. Fortet et B. Mourier, Convergence de la répartition empirique vers la répartition théorétique, Ann. Sci. École Norm. Sup. 70 (1953), 267-285.

[9] R. Isaac, Markov processes and unique stationary probability measures, Pacific J. Math. 12 (1962), 273-286.

[10] A. Lasota and M. C. Mackey, Chaos, Fractals and Noise. Stochastic Aspects of Dynamics, Springer, 1994.

[11] A. Lasota and J. Myjak, Semifractals on Polish spaces, Bull. Polish Acad. Sci. Math. 46 (1998), 179-196.

[12] A. Lasota and J. A. Yorke, Lower bound technique for Markov operators and iterated function systems, Random Comput. Dynam. 2 (1994), 41-77.

[13] K. Łoskot and R. Rudnicki, Limit theorems for stochastically perturbed dynamical systems, J. Appl. Probab. 32 (1995), 459-469.

[14] G. Lu and A. Mukherjea, Invariant measures and Markov chains, Probab. Math. Statist. 17 (1997), 115-138.

[15] S. P. Meyn and R. L. Tweedie, Markov Chains and Stochastic Stability, Springer, 1993. 
[16] R. Sine, Convergence theorems for weakly almost periodic Markov operators, Israel J. Math. 19 (1974), 246-255.

[17] Ö. Stenflo, Iterated function systems controlled by a semi-Markov chain, Theory Stochastic Processes 18 (1996), 305-313.

[18] T. Szarek, Markov operators acting on Polish spaces, Ann. Polon. Math. 67 (1997), $247-257$.

[19] - , The stability of Markov operators on Polish spaces, Studia Math. 143 (2000), $145-152$.

Institute of Mathematics

Polish Academy of Sciences

Bankowa 14

40-007 Katowice, Poland

E-mail: szarek@gate.math.us.edu.pl

Received July 19, 2001

Revised version June 24, 2002 